\title{
Quantitative image analysis of the MIB-1 proliferation index in Non-Hodgkin Lymphoma
}

Nairi Tchrakian ${ }^{*}$, Tatjana Nehhozina², Greg Lee ${ }^{2}$, Julie McFadden'1, Susan Russell', Larry Bacon², Elisabeth Vandenberghe² and Richard Flavin ${ }^{1}$

*Correspondence: nairitchrakian@gmail.com

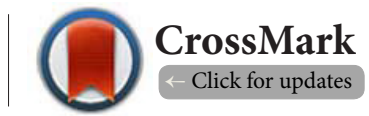

'Departments of Histopathology, St James's Hospital, Dublin 8, Ireland.

${ }^{2}$ Departments of Haematology, St James's Hospital, Dublin 8, Ireland.

\begin{abstract}
Background: MIB-1 is a useful prognostic tool in Non-Hodgkin Lymphoma (NHL); in general, a high proliferative index (PI) is associated with aggressive disease. Quantitative image analysis (QIA) of tumour biomarkers has become more widespread due to the impractical nature of manual counting and the interobserver variability associated with semi-quantitative analysis. We hypothesized that a QIA algorithm would give a more accurate PI estimate, leading to better overall survival associations in cases of NHL.

Methods: 51 MIB-1 immunohistochemical slides from NHL cases were scanned using Virtuoso image analysis software (Roche Tissue Diagnostics/Ventana), and the PI was digitally quantified using a nuclear algorithm from selected regions of interest. The diagnostic PI quantified by the pathologist was recorded from the original report and validated by both study pathologists. Medical records were reviewed and disease progression details and survival time noted.

Results: Quantification of the MIB-1 index by image analysis showed strong positive correlation with the semi-quantified value(Spearman correlation coefficient $=0.78 ; \mathrm{p}<0.0001)$. Digitally-quantified MIB1 proliferation indices demonstrated significance with overall survival in the lymphoma cohort (HR 1.6 (0.2-10.6); $\mathrm{p}=0.05)$ when compared with the semi-quantified values (HR 1.6 (0.2-10.6); $\mathrm{p}=0.6)$. Similarly digitally-quantified MIB-1 proliferation indices demonstrated significance with progression free survival in the lymphoma cohort (HR 2.8 (0.6-13.5); $\mathrm{p}=0.05)$ when compared with the semi-quantified values (HR 0.4 $(0.2-1.2) ; \mathrm{p}=0.1)$.

Conclusions: QIA is a useful, reproducible and objective method of determining the MIB-1 PI in NHL cases and consideration should be given to introducing computer based algorithms into routine clinical haematopathology practice. However, QIA validation should be assessed in a larger sample cohort before consideration is given to introducing computer-based algorithms into routine clinical pathology practice.
\end{abstract}

Keywords: lymphoma, MIB-1, proliferation index, quantitative image analysis, prognosis

\section{Introduction}

MIB-1 is a monoclonal antibody directed against the nuclear protein Ki-67, which is expressed in all phases of the cell cycle except G0 [1]. For this reason it is used as a cellular marker for proliferation; the presence of nuclear staining indicates an active cycling cell.Having gained widespread use as a proliferation marker, MIB-1 has been shown to be a useful prognostic tool in several lymphoma subtypes. Numerous studies indicate that a high proliferation rate is associated with a more aggressive disease course, and hence a poorer prognosis [2-6].
In routine clinical practice, haematopathologists often adopt a semi-quantitative approach to assess the proliferation index. This generally involves visually scanning representative tumour foci on the glass slide and estimating the number of positive tumour nuclei, by performing a formal cell count. There is some debate over whether this estimation is sufficient, as it is susceptible to subjectivity and high rates of inter-observer variability and can be both laborious and time-consuming.

In recent years, reliable and reproducible digital analytical techniques have been developed, rendering software-automat-

(c) 2018 Tchrakian et al; licensee Herbert Publications Ltd. This is an Open Access article distributed under the terms of Creative Commons Attribution License (http://creativecommons.org/licenses/by/3.0). This permits unrestricted use, distribution, and reproduction in any medium, provided the original work is properly cited. 
ed counting of cells via quantitative image analysis a viable option. Indeed, image analysis is becoming increasingly more widespread for quantification of biomarkers in tumours, with prominent usage of whole-slide imaging systems in research applications in particular $[\mathbf{7 , 8}]$. Importantly, automated image analysis has the potential to yield continuous variable data, unlike semi-quantitative analysis, which typically furnishes data in discrete increments. Many studies have shown digital quantification methods to demonstrate comparable accuracy to carefully performed formal manual quantification $[4,9,10]$. In this way, quantitative image analysis has the potential to objectively, accurately and consistently provide biomarker labelling indices, both in research and clinical settings [11].

In this study, we hypothesized that a quantitative image analysis algorithm would provide a more accurate estimate of the MIB-1 proliferation index in cases of Non-Hodgkin Lymphoma $(\mathrm{NHL})$ rather than traditional semi-quantitative analysis.

\section{Materials and methods Case selection}

The histopathology database at St James's Hospital, Dublin, was searched between January 2008 and December 2012 (inclusive) for cases of NHL with available MIB-1 immunohistochemistry, and the diagnoses were reviewed and reclassified according to World Health Organisation (WHO) 2016 criteria. These cases were unbiased with regard to treatment. Rather than focusing on specific histotypes of lymphoma, as proof of principle, 51 cases of $\mathrm{NHL}$ (from 49 patients) were randomly selected, diagnoses reviewed and classified according to World Health Organisation (WHO) 2008 criteria. Cases which presented technical difficulties in the estimation of the MIB-1 proliferation index (i.e. due to poor fixation, extensive necrosis, artefact etc.) were excluded from the study. The study cohort included: diffuse large B-cell lymphoma (DLBCL; $\mathrm{n}=24$ ); follicular lymphoma ( $\mathrm{FL}$; $\mathrm{n}=21$; mantle cell lymphoma (MCL; $\mathrm{n}=4)$; B-cell lymphoma with features intermediate between DLBCL and Burkitt lymphoma $(n=2)$ (Figure 1). Patients' medical records were reviewed and clinical data collected, including date of diagnosis, type of biopsy, site of biopsy, therapy received, details of relapse (if any), date of death (if deceased), and overall survival time (defined as time from initial diagnosis until death). The study was approved by the SJH/AMNCH (St James's Hospital/Adelaide and Meath Hospital, Dublin, incorporating the National Children's Hospital) Research Ethics Committee.

\section{Ki-67 Immunohistochemistry}

Ki-67 immunohistochemistry was performed on $4 \mu \mathrm{m}$-thick formalin-fixed paraffin-embedded tissue sections using the Refine Polymer Kit on the Leica Bond Max Autostainer. The primary antibody used was the Dako antibody for the Ki-67 clone MIB-1 (M7240) with an incubation time of 15 minutes at room temperature, at 1:80 dilution. Antibody retrieval was

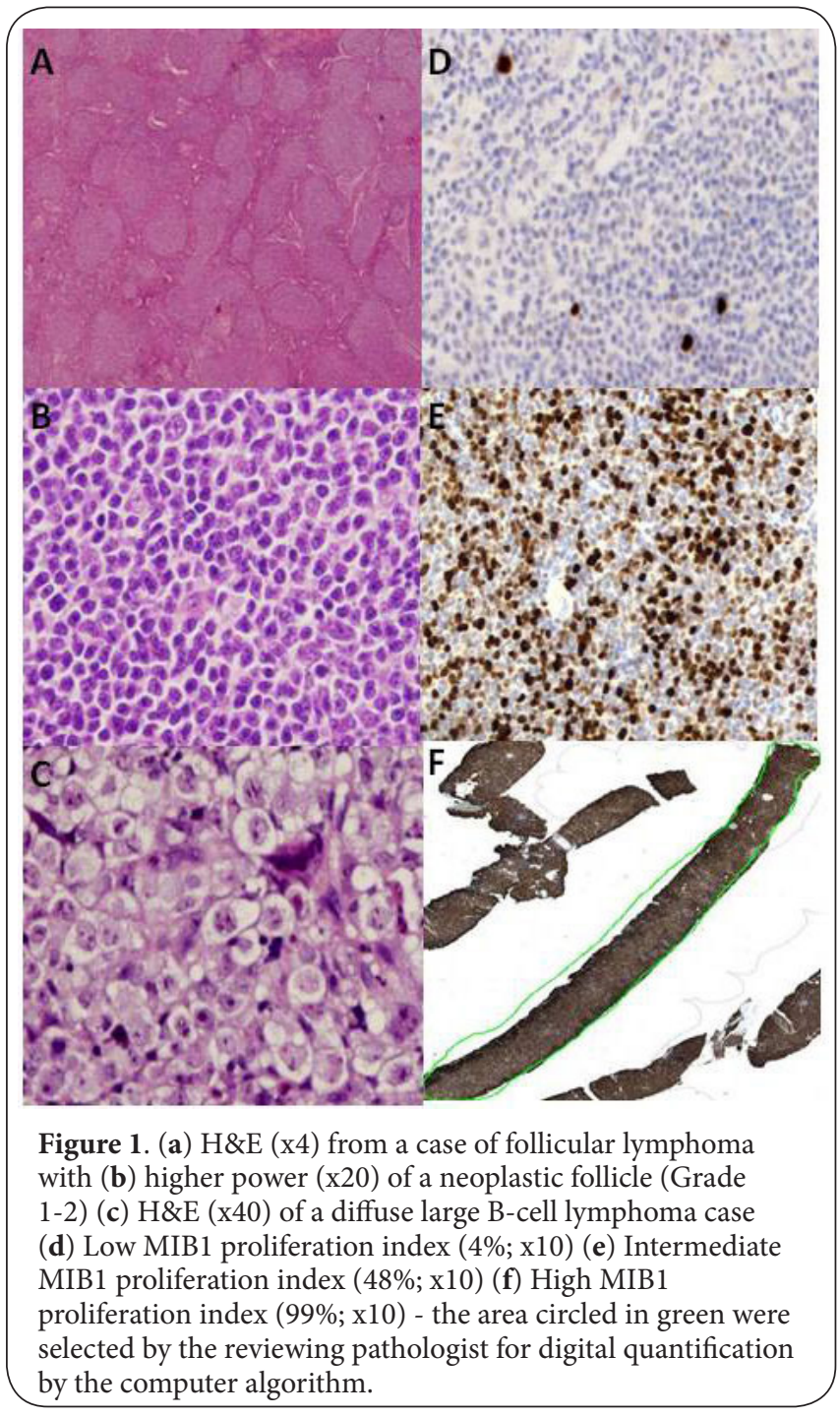

performed with ER2 (epitope retrieval solution) for 30 minutes.

\section{Ki-67 analysis}

The MIB-1 proliferation index semi-quantitatively assessed by the pathologist at diagnosis was recorded from the original report and validated by both study pathologists by performing a formal count of 200 tumour nuclei followed by averaging of scores (as per a previously reported protocol for Mantle cell lymphoma) [6]. If the index was given as a range (e.g. 60$70 \%$ ), the mean value (65\% in the example given) was used for the statistical analysis.

MIB-1 immunohistochemical slides were scanned at 20x magnification using Virtuoso image analysis software (Roche Tissue Diagnostics/Ventana ${ }^{\mathrm{TM}}$ ) and a Roche Ventana iScan Coreo platform. The scanned images were uploaded onto a digital slide repository (Figure 1). Regions of interest (ROI) of the NHL were manually selected by one of the study pathologists for analysis, excluding areas of poor fixation, crush 
artefact, necrosis, residual germinal centres and foci where there appeared to be a significant number of background reactive cells. In the cases of follicular lymphoma, neoplastic follicles were preferentially selected for analysis. The MIB-1 index was digitally quantified using a nuclear algorithm from the selected ROI based on the differentiation between brown (positive) and blue (counter) staining. Total percentage nuclear positivity was recorded for each case.

\section{Statistical analysis}

Spearman's correlation coefficient and linear regression analysis were used to correlate semi-quantified and digitally quantified MIB-1 indices using Microsoft Analyse-It ${ }^{\oplus}$. MedCalc Version 13.3.3 was used to obtain Kaplan-Meier estimates of one and five-year survival rates, which were then compared using the log-rank test.

\section{Results \\ Clinical data}

For thirty-four of the forty-nine patients $(34 / 49 ; 69 \%)$ (Table 1) enlisted at the beginning of the study, sufficient clinical follow-up data was available for further statistical analysis.

Table 1. Demographic and clinical characteristics of the cohort of 34 patients who had MIB-1 digital quantification performed and who were suitable for survival analysis.

\begin{tabular}{llll}
\hline Characteristic & $\begin{array}{l}\text { DiffuseLarge } \\
\text { B-cell Lymphoma }\end{array}$ & $\begin{array}{l}\text { Follicular } \\
\text { Lymphoma }\end{array}$ & Total \\
\hline $\begin{array}{l}\text { Number of Patients } \\
\text { Gender }\end{array}$ & $18(53 \%)$ & $16(47 \%)^{*}$ & $34(100 \%)$ \\
$\begin{array}{l}\text { Male } \\
\text { Female }\end{array}$ & $9(26 \%)$ & $6(18 \%)$ & $15(44 \%)$ \\
$\begin{array}{l}\text { Relapse Status } \\
\text { Yes }\end{array}$ & $9(26 \%)$ & $10(30 \%)$ & $19(56 \%)$ \\
No & $10(29 \%)$ & $5(15 \%)$ & $15(44 \%)$ \\
$\begin{array}{l}\text { Outcome } \\
\text { DOD }\end{array}$ & $8(24 \%)$ & $11(32 \%)$ & $19(56 \%)$ \\
AWD & $5(15 \%)$ & 0 & $5(15 \%)$ \\
NED & $4(12 \%)$ & $11(32 \%)$ & $15(44 \%)$ \\
\hline
\end{tabular}

DLBCL=Diffuse Large B-Cell Lymphoma;

$\mathrm{FL}=$ Follicular Lymphoma;

DOD: dead of disease;

AWD: alive with disease; NED: no evidence of disease

* Grade 1-2=14/16 (87.5\%), Grade 3=2/16 (12.5\%)

\section{Diffuse Large B-cell Lymphoma}

The male to female ratio in this group was $1: 1$, and the mean age of diagnosis was 52 (median: 50; range: 27-87). Ten of the eighteen $(10 / 18 ; 56 \%)$ patients relapsed; the mean time to recurrence was 26 months. In three patients $(3 / 18 ; 17 \%)$, stem cell transplantation (SCT) was undertaken; one autologous and two sibling allogeneic subtypes. These were performed an average 42 months after the initial diagnosis of lymphoma (range $=20-80$ months). Post $-\mathrm{SCT}$ relapse was recorded in one of these three cases (33\%); here, relapse occurred nine months after myeloablative allogeneic SCT. Five patients $(5 / 18$;
$28 \%$ ) died of disease at the time of follow-up. No evidence of lymphoma was recorded in nine of the remaining thirteen surviving individuals (69\%). The four remaining cases of the thirteen surviving patients (31\%) were reported to be 'alive with disease'.

\section{Follicular lymphoma}

The male to female ratio in this group was 3:5, and the mean age of diagnosis was 60 (median: 61; range: 39-72). Five of the sixteen $(5 / 16 ; 31 \%)$ patients relapsed; the mean time to recurrence was 39 months. In one patient $(1 / 16 ; 6 \%)$, stem cell transplantation (SCT) was undertaken (autologous subtype). This was performed 94 months after the initial diagnosis of lymphoma. Post-SCT relapse did not occur. No patients (0\%) had died of disease at the time of follow-up. No evidence of lymphoma was recorded in five of the sixteen surviving individuals (31\%). The eleven remaining patients (69\%) were reported to be 'alive with disease'.

\section{Correlation of Digitally Quantified and Semi-Quantified MIB-1 Indices}

Fifty-one MIB-1 immunohistochemical slides obtained from 49 patients with NHL were retrieved from the archives. 41/51 cases $(80 \%)$ were successfully scanned and digitally quantified. The digital quantification software consistently yielded a higher MIB-1 proliferation index than that semi-quantified by the study pathologist. Mean semi-quantified MIB-1 index was $49 \%$ in comparison to $72.17 \%$ by digital evaluation. For cases where the reported MIB-1 index was between $10 \%$ and $90 \%$, the mean semi-quantified and digitally-quantified values were $49.86 \%$ and $75.90 \%$, respectively (Spearman correlation coefficient $=0.7407, p<0.0001$ ). In keeping with the nature of semi-quantitative analysis, the pathologist's estimate tended to be reported in increments of 5 or 10 percentage points, while the digitally-quantified values were reported with greater precision to the nearest centile. Nonetheless, there was a strong positive correlation of the digitally quantified and semi-quantified value(Spearman correlation coefficient $=0.78$; $p<0.0001 ; R^{2}=0.64, p<0.0001$ ) (Figure 2).

\section{MIB-1 proliferation index and survival analysis}

The MIB-1 indices obtained by both analysis modalities were compared with overall patient survival. In this cohort of thirtyfour patients, the mean semi-quantified MIB-1 index was $46.21 \%$ in comparison to the mean value of $70.03 \%$ obtained by digital evaluation $(p<0.0001)$. For both analysis modalities, one- and five-year survival rates were $89.8 \%$ and $76.3 \%$, respectively. Digitally-quantified MIB-1 proliferation indices demonstrated significance with overall survival in the lymphoma cohort (HR $1.6(0.2-10.6) ; p=0.05)$ when compared with the semi-quantified values (HR 1.6 (0.2-10.6); $p=0.6)$-Figure 3 . Similarly digitally-quantified MIB-1 proliferation indices demonstrated significance with progression free survival in the lymphoma cohort (HR 2.8 (0.6-13.5); $\mathrm{p}=0.05)$ when compared 


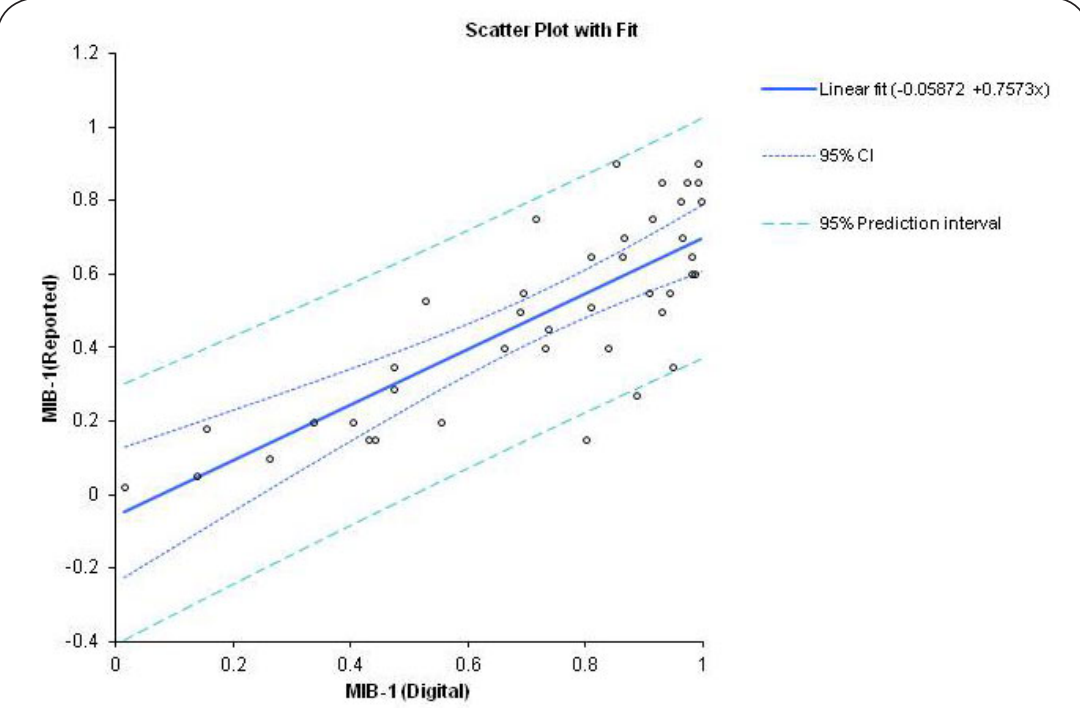

Figure 2. Scatter Plot with fit showing the strong positive correlation between digitally-quantified and reported MIB- 1 proliferation indices $\left(\mathrm{R}^{2}=0.64\right.$; $\mathrm{p}<0.0001)$.

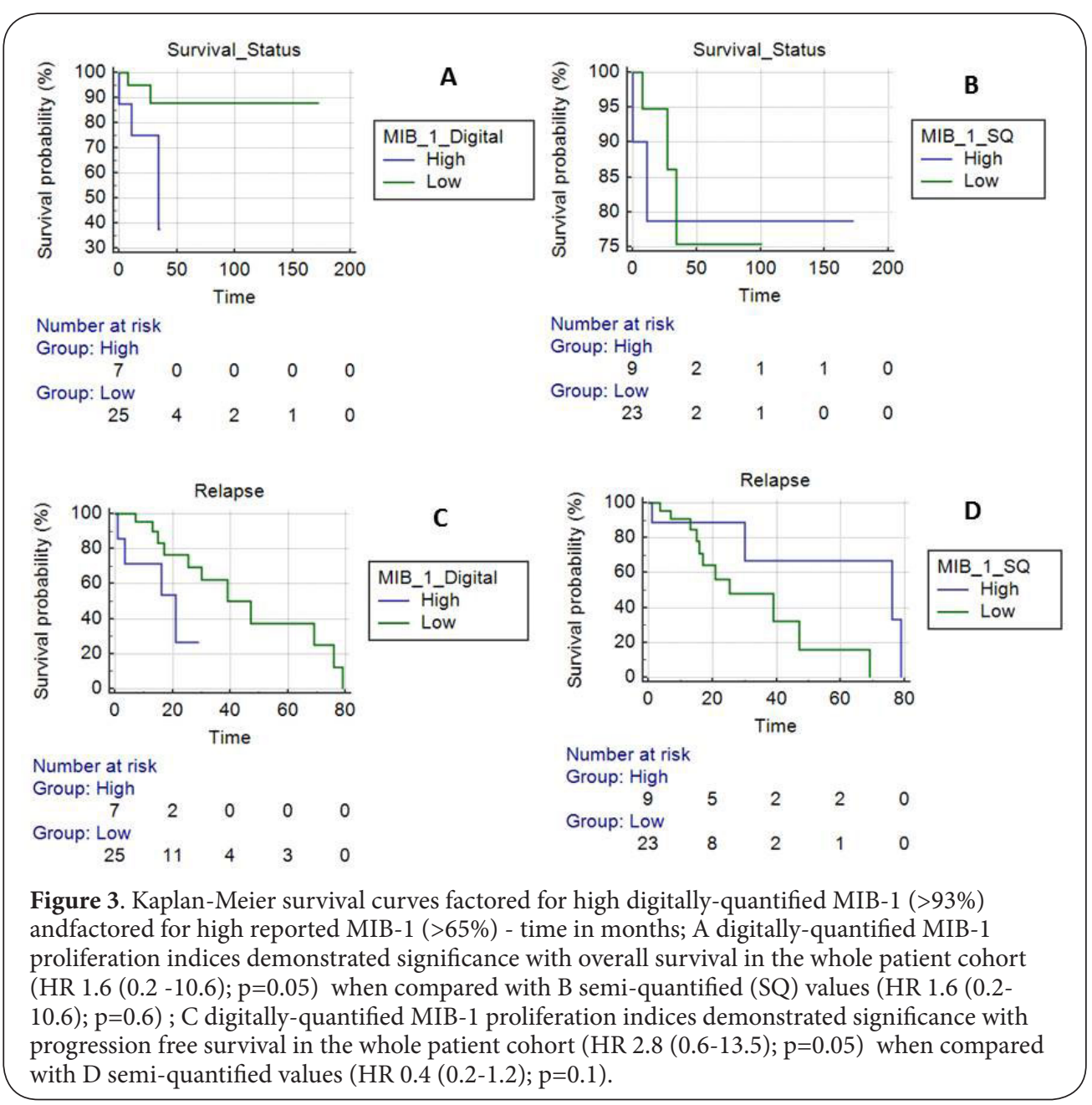


with the semi-quantified values (HR 0.4 (0.2-1.2); $\mathrm{p}=0.1$ )-see Figure 3. MIB-1 proliferation indices were not associated with progression free or overall survival for both semi-quantified and digital values for individual subgroups of $D L B C L$ and $F L$ (data not shown).

\section{Discussion}

In this study, we show that digital quantification of the MIB-1 proliferation index correlates strongly with semi-quantified values, demonstrating that quantitative image analysis provides an accurate and reproducible means to quantify the $\mathrm{Ki}-67$ index in cases of NHL. Furthermore we demonstrate a significant association between digitally quantified Ki-67 index and progression and overall survival when compared to semi-quantified values indicating that QIA may be a useful and objective method of determining the MIB-1 PI in NHL cases.

Because of the very close correlation between digitallyquantified and semi-quantified MIB-1 proliferation indices, a cautious approach must be taken in introducing computerbased algorithms in routine clinical haematopathology. Digital quantification does after all require extra equipment, greater expense, and is significantly more time-consuming compared with traditional semi-quantitative estimation of the MIB-1 index. Computerised image analysis requires prior digitisation of microscopic images with digital slide scanners. An automated image analysis programme then detects and quantifies individual immunostained cells based on their size, shape and colour. This, however, may prove to be difficult in tissue with densely-packed nuclei, such as lymphoma. In these cases, sophisticated algorithms are required to interpret such features as tumour cell overlap. In spite of this sophistication, digital quantification systems generally still require some user interaction in order to adequately select regions of the slide for analysis [12]. Furthermore, various technical issues may impinge on the utility of the MIB-1 proliferative index in lymphoma. Van der Laak et al (2012) [10] suggest that to use MIB-1 routinely as a prognostic marker, attention must be given to standardising immunohistochemical staining procedures; they state that consistency of staining may be compromised by delayed fixation and type of fixation. Another potential limitation of the proliferative rate as measured by MIB- 1 is that many different antibodies are commercially available, and these may differ in their efficacy.

Selection of a representative area of tumour for quantification of the MIB-1 proliferative index in NHL may present a significant element of subjectivity. It is possible that confusion exists among pathologists as to which area of the tumour should be selected for analysis. As previously noted, we aimed to select areas for analysis which were representative of the entire volume of tumour on the slide. In the cases of follicular lymphoma, neoplastic follicles were selected. Another important point to consider is that with a relatively large tumour volume, a global assessment of the MIB-1 index in a given case may not be possible. This assumes especial relevance where focal high-grade transformation is present and will affect the biological behaviour of the tumour. ChabotRichards et al examined the prognostic significance of the MIB-1 proliferation index in Diffuse Large B-Cell Lymphoma and showed no correlation with outcome [2]. The authors postulated that strong associations reported previously in other studies may have been affected by biases, including the deliberate selection of areas of higher proliferation. It may be that the variability between pathologists in selecting the area of the tumour for analysis weakens the association between the MIB-1 index and prognosis. Naturally, areas of intact tumour should be prioritised for analysis, and areas of necrosis, crush artefact and poor fixation should be avoided.

A few studies have examined the potential use of quantitative image analysis to measure the MIB-1 proliferation index in $\mathrm{NHL}$ and its utility in assessing prognosis. Conclusions vary regarding the correlation between the digitally-quantified proliferative index and the semi-quantified indices. Furthermore, some studies examining the prognostic utility of the MIB-1 proliferative index in lymphoma suggest that a higher proliferative index heralds more aggressive disease with a poor prognosis [13]. However, a significant number of studies show conflicting data, asserting that there may be either no association between the two (similar to this study), $[\mathbf{2}, \mathbf{1 4 , 1 5}]$ or, indeed, that the association is reversed [1]. Klapper et al did not find a convincing concordance between both semi-quantification and quantitative image analysis of the MIB-1 proliferation index and the modality they took as the gold standard; namely, manual counting [7]. They suggested that in the case of Mantle Cell Lymphoma, there may be a failure on the part of the digital quantification systems to recognise, and hence to quantify the densely packed, relatively small neoplastic cells that characterise the disease. They advised that in selecting areas of the slide for analysis, only representative areas should be used, which do not include residual germinal centres, 'hot-spots' of proliferation, or background reactive T-cells. The authors also noted that digitally-obtained MIB-1 indices might correlate better with clinical outcome than the manually-counted value, but that further studies with larger sample sizes will be necessary to ascertain this. Notably, a recent study by Samols et al [4] examining follicular lymphoma showed that although a higher proliferative index in general correlated with a higher histologic grade, the authors identified a subset of low grade follicular lymphoma with a high proliferative index, a finding which itself had been previously reported by Wang et al (2005) [16]. This cohort reportedly showed a shorter overall survival, and Samols et al suggested that they should be considered as a different prognostic group compared with patients with follicular lymphoma whose proliferative fraction is low. In cases of follicular lymphoma, the neoplastic follicles are selected out for analysis, rather than the relatively quiescent interfollicular areas, as advised in a 2006 paper by Zhang et al on quantitative image analysis in $\mathrm{FL}$ [9]. 
There are a number of limitations to this pilot study. The sample size is small and assessing a mixed cohort of both follicular lymphoma and DLBCL in the survival analysis has introduced a bias as both lymphoma subtypes have differing biological behaviour and both are treated differently. There may also have been staining variability as the Ki- 67 staining was performed over a range of time. In conclusion, QIA is a useful, reproducible and objective method of determining the MIB-1 PI in NHL; the MIB-1 PI in NHL; however, it needs to be validated in a larger cohort of lymphoma samples before consideration is given to introducing computer based algorithms into routine clinical haematopathology practice.

\section{Competing interests}

The authors declare that they have no competing interests.

Authors' contributions

\begin{tabular}{|l|c|c|c|c|c|c|c|c|}
\hline Authors' contributions & NT & TN & GL & JM & SR & LB & EV & RF \\
\hline Research concept and design & -- & -- & -- & -- & -- & -- & -- & $\checkmark$ \\
\hline Collection and/or assembly of data & $\checkmark$ & -- & $\checkmark$ & $\checkmark$ & $\checkmark$ & -- & -- & -- \\
\hline Data analysis and interpretation & -- & $\checkmark$ & -- & -- & -- & -- & -- & $\checkmark$ \\
\hline Writing the article & $\checkmark$ & -- & -- & -- & -- & -- & -- & $\checkmark$ \\
\hline Critical revision of the article & -- & -- & -- & -- & -- & $\checkmark$ & $\checkmark$ & $\checkmark$ \\
\hline Final approval of article & -- & -- & -- & -- & -- & -- & -- & $\checkmark$ \\
\hline Statistical analysis & -- & $\checkmark$ & -- & -- & -- & -- & -- & $\checkmark$ \\
\hline
\end{tabular}

\section{Acknowledgements}

We would like to thank Roche Diagnostics Limited (Laura Daly and Louise Flynn, in particular) and the RTD Applications Laboratory at Roche Diagnostics Limited for scanning the MIB-1 immunohistochemical slides and for providing the technical equipment and advice necessary for the subsequent digital quantification.

\section{Publication history}

EIC: Gaetano Giuseppe Magro, University of Catania, Italy. Received: 23-Nov-2017 Final Revised: 29-Dec-2017

Accepted: 06-Jan-2018 Published: 26-Jan-2018

\section{References}

1. Hasselblom S, Ridell B, Sigurdardottir M, Hansson U, Nilsson-Ehle H and Andersson PO. Low rather than high Ki-67 protein expression is an adverse prognostic factor in diffuse large B-cell lymphoma. Leuk Lymphoma. 2008; 49:1501-9. | Article | PubMed

2. Chabot-Richards DS, Martin DR, Myers OB, Czuchlewski DR and Hunt KE. Quantitative image analysis in the assessment of diffuse large B-cell lymphoma. Mod Pathol. 2011; 24:1598-605. I Article I PubMed Abstract | PubMed FullText

3. Koster A, Tromp HA, Raemaekers JM, Borm GF, Hebeda K, Mackenzie MA and van Krieken $\mathrm{JH}$. The prognostic significance of the intra-follicular tumor cell proliferative rate in follicular lymphoma. Haematologica. 2007; 92:184-90. | Article | PubMed

4. Samols MA, Smith NE, Gerber JM, Vuica-Ross M, Gocke CD, Burns KH, Borowitz MJ, Cornish TC and Duffield AS. Software-automated counting of Ki-67 proliferation index correlates with pathologic grade and disease progression of follicular lymphomas. Am J Clin Pathol. 2013; 140:579-87. | Article | PubMed Abstract | PubMed FullText

5. Tiemann M, Schrader C, Klapper W, Dreyling MH, Campo E, Norton A, Berger F, Kluin P, Ott G, Pileri S, Pedrinis E, Feller AC, Merz H, Janssen D, Hansmann ML, Krieken H, Moller P, Stein H, Unterhalt M, Hiddemann $\mathrm{W}$ and Parwaresch R. Histopathology, cell proliferation indices and clinical outcome in 304 patients with mantle cell lymphoma (MCL): a clinicopathological study from the European MCL Network. Br J Haematol. 2005; 131:29-38. | Article | PubMed

6. Vogt $\mathrm{N}$ and Klapper W. Variability in morphology and cell proliferation in sequential biopsies of mantle cell lymphoma at diagnosis and relapse: clinical correlation and insights into disease progression Histopathology. 2013; 62:334-42. | Article | PubMed

7. Klapper W, Hoster E, Determann O, Oschlies I, van der Laak J, Berger F, Bernd HW, Cabecadas J, Campo E, Cogliatti S, Hansmann ML, Kluin PM, Kodet R, Krivolapov YA, Loddenkemper C, Stein H, Moller P, Barth TE, Muller-Hermelink K, Rosenwald A, Ott G, Pileri S, Ralfkiaer E, Rymkiewicz $\mathrm{G}$, van Krieken JH, Wacker HH, Unterhalt M, Hiddemann W and Dreyling $\mathrm{M}$. Ki-67 as a prognostic marker in mantle cell lymphoma-consensus guidelines of the pathology panel of the European MCL Network. J Hematop. 2009; 2:103-11. | Article | PubMed Abstract | PubMed FullText

8. Thrall MJ, Wimmer $\mathrm{JL}$ and Schwartz MR. Validation of multiple whole slide imaging scanners based on the guideline from the College of American Pathologists Pathology and Laboratory Quality Center. Arch Pathol Lab Med. 2015; 139:656-64. | Article | PubMed

9. Zhang K, Prichard JW, Yoder S, De J and Lin F. Utility of SKP2 and MIB-1 in grading follicular lymphoma using quantitative imaging analysis. Hum Pathol. 2007; 38:878-82. | Article | PubMed

10. van der Laak JA, van Engelen N, Melissen M and Hebeda KM. Automated measurement of MIB-1 positive area as an alternative to counting in follicular lymphoma. Cytometry A. 2012; 81:527-31. | Article | PubMed

11. Schaffel R, Hedvat CV, Teruya-Feldstein J, Persky D, Maragulia J, Lin D, Portlock CS, Moskowitz CH and Zelenetz AD. Prognostic impact of proliferative index determined by quantitative image analysis and the International Prognostic Index in patients with mantle cell lymphoma. Ann Oncol. 2010; 21:133-9. | Article | PubMed Abstract | PubMed FullText

12. Lejeune M, Jaen J, Pons L, Lopez C, Salvado MT, Bosch R, Garcia M, Escriva P, Baucells J, Cugat $X$ and Alvaro T. Quantification of diverse subcellular immunohistochemical markers with clinicobiological relevancies: validation of a new computer-assisted image analysis procedure. J Anat. 2008; 212:868-78. | Article | PubMed Abstract | PubMed FullText

13. Ali AE, Morgen EK, Geddie WR, Boerner SL, Massey C, Bailey DJ and da Cunha Santos G. Classifying B-cell non-Hodgkin lymphoma by using MIB-1 proliferative index in fine-needle aspirates. Cancer Cytopathol. 2010; 118:166-72. | Article | PubMed

14. Czader M, Mazur J, Pettersson M, Liliemark J, Stromberg M, Christensson B, Tribukait B, Auer G, Ost A and Porwit A. Prognostic significance of proliferative and apoptotic fractions in low grade follicle center cellderived non-Hodgkin's lymphomas. Cancer. 1996; 77:1180-8. | Article | PubMed

15. Zhang A, Ohshima K, Sato K, Kanda M, Suzumiya J, Shimazaki K, Kawasaki $\mathrm{C}$ and Kikuchi M. Prognostic clinicopathologic factors, including immunologic expression in diffuse large B-cell lymphomas. Pathol Int. 1999; 49:1043-52. | Article | PubMed

16. Wang SA, Wang L, Hochberg EP, Muzikansky A, Harris NL and Hasserjian RP. Low histologic grade follicular lymphoma with high proliferation index: morphologic and clinical features. Am J Surg Pathol. 2005; 29:1490-6. | Article | PubMed

\section{Citation:}

Tchrakian N, Nehhozina T, Lee G, McFadden J, Russell S, Bacon L, Vandenberghe E and Flavin R. Quantitative image analysis of the MIB-1 proliferation index in Non-Hodgkin Lymphoma. J Histol Histopathol. 2018; 5:3. http://dx.doi.org/10.7243/2055-091X-5-3 